\title{
Interpolating Nonadiabatic Molecular Dynamics Hamiltonian with Artificial Neural Networks
}

\author{
Bipeng Wang, Weibin Chu, Alexandre Tkatchenko, and Oleg V. Prezhdo* \\ Cite This: J. Phys. Chem. Lett. 2021, 12, 6070-6077 \\ Read Online
}

ACCESS

Lلll Metrics \& More

Article Recommendations

Supporting Information

ABSTRACT: Nonadiabatic (NA) molecular dynamics (MD) allows one to study farfrom-equilibrium processes involving excited electronic states coupled to atomic motions. While NAMD involves expensive calculations of excitation energies and NA couplings (NACs), ground-state properties require much less effort and can be obtained with machine learning (ML) at a fraction of the ab initio cost. Application of ML to excited states and NACs is more challenging, due to costly reference methods, many states, and complex geometry dependence. We developed a NAMD methodology that avoids time extrapolation of excitation energies and NACs. Instead,

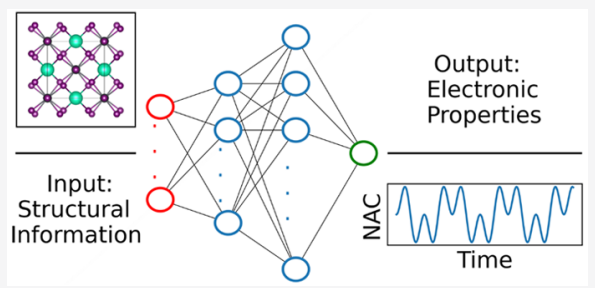
under the classical path approximation that employs a precomputed ground-state trajectory, we use a small fraction (2\%) of the geometries to train neural networks and obtain excited-state energies and NACs for the remaining $98 \%$ of the geometries by interpolation. Demonstrated with metal halide perovskites that exhibit complex MD, the method provides nearly two orders of computational savings while generating accurate NAMD results.

$\mathrm{N}$ onadiabatic (NA) molecular dynamics (MD) is a powerful computational tool that allows one to model excited-state processes in a broad range of chemical systems. ${ }^{1-3}$ Such simulations are able to mimic mostly closely, at the atomistic level and in the time domain, numerous timeresolved spectroscopy experiments, many of which are now routinely used to characterize ultrafast response of molecules and materials to external electromagnetic fields. ${ }^{4-6}$ Such experimental and theoretical studies form the basis for many modern-day applications, including solar cells, light-emitting diodes, field-effect transistors, sensors, quantum information devices, etc. NAMD simulation requires knowledge of geometry-dependent energies and forces for ground and excited states, and NA coupling (NAC) between the states. Such information is most commonly obtained by ab initio electronic structure calculations performed with system geometries along $\mathrm{MD}$ trajectories. The calculations are computationally demanding, especially those involving the excited state and NAC. Therefore, efforts to avoid the need to perform thousands of electronic structure calculations can provide great computational savings. Rooted in the fact that $\mathrm{MD}$ in many condensed matter and nanoscale systems are weakly dependent on the occupied electronic state and are driven by thermal fluctuations, the classical path approximation (CPA) replaces multiple excited-state trajectories with a single ground-state trajectory, greatly simplifying the NAMD calculations. $^{7-9}$ Still, even ground-state $\mathrm{MD}$ trajectories are expensive to obtain at the ab initio level for large, nanoscale systems and sufficiently long time scales that may involve, for instance, formation of polarons ${ }^{10}$ or diffusion of defects. ${ }^{11}$

Development of force fields (FF) to replace ab initio calculations and obtain ground-state $\mathrm{MD}$ trajectories has a long history, starting from very simple models, e.g., harmonic and Lennard-Jones potentials, to reactive $\mathrm{FF}^{12}$ and most recently, to FF developed using machine learning $(\mathrm{ML})$ techniques, ${ }^{13,14}$ such as artificial neural networks (ANNs). Analogous development of parametrized models for advanced electronic properties, ${ }^{15-17}$ including excited-state forces and NAC, ${ }^{18-20}$ has been limited, because $a b$ initio calculations required for the parametrization are expensive, and the corresponding experimental data are scarce. Moreover, NAC has a notably more complex dependence on system geometry than energy and force, and the number of NAC matrix elements scales quadratically with the number of electronic states. The ability of ANNs to represent essentially any set of data, rooted in the universal approximation theorem, ${ }^{21}$ and their current rapid development and popularity in all areas of science and engineering raise the prospects that not only energies and forces but also NACs can be efficiently parametrized. Several efforts have been reported in this regard, ${ }^{18-20}$ drawing parallels with ML FF development; ${ }^{13}$ i.e., a set of geometries is used to compute NAC and train an ANN, and then the ANN predicts NACs for future geometries. Because NAC is a more complex property than energy, such a strategy is quite challenging, requiring additional ANN layers and more thorough training.

Received: May 24, 2021

Accepted: June 23, 2021 
In comparison, ground-state FFs are already commonly generated using ML techniques. Thus, long ab initio quality ground-state trajectories can be obtained. Rather than attempting to extrapolate excitation energies and NAC in a similar way, we propose to sample these properties along a pregenerated trajectory and then interpolate to obtain the values for the intermediate geometries. Such a strategy cannot be used in a general NAMD simulation since an NAMD trajectory cannot be known a priori. However, the strategy can provide huge computational savings if NAMD is performed under the $\mathrm{CPA},{ }^{7-9,14}$ i.e., if the trajectory is precomputed because $\mathrm{MD}$ is driven by thermal fluctuations rather than differences in excited and ground-state forces. Such a situation is common to many classes of materials and processes. ${ }^{22-30}$

In this letter, we develop a novel strategy for predicting excitation energies and NACs for NAMD simulation using ML. Given a trajectory, which can be precomputed using a ML FF, we sample excitation energies and NAC for a small fraction (2\%) of points along the trajectory and employ ANNs to obtain the missing values for the remaining points. The strategy allows us to reduce the computational cost of ab initio calculations of excited-state energies and NACs under the CPA by nearly two orders of magnitude, while obtaining reliable NAMD results in good agreement with the full ab initio calculations. We demonstrate the method with metal halide perovskites (MHPs), which are among the most popular modern-day materials for solar energy and optoelectronic applications. Easy to manufacture, MHPs possess many unusual physical and chemical properties, combining those of inorganic solids, organic matter, and even liquids. They exhibit complicated $\mathrm{MD}$ with a broad range of anharmonic motions and time scales, creating challenges even for ML FF development. The developed ML-NAMD method works very well in this case, including both a pristine MHP and a MHP with a common defect that creates a midgap charge trap state.

MHPs have gained a lot of attention recently because of their low cost and ability to convert solar energy to electricity in a clean and sustainable way, as a viable alternative to fossil fuels. A very large number of studies are currently reported on hybrid organic-inorganic and all-inorganic MHPs. They can be both $\mathrm{Pb}$-based, as introduced initially, ${ }^{31,32}$ and non- $\mathrm{Pb}$ based to avoid toxicity associated with $\mathrm{Pb}$ atoms. ${ }^{33}$ Both $3 \mathrm{D}$ and 2D MHPs of broadly varying compositions have been produced and extensively studied. Discovered in the 19th century in the Ural Mountains, perovskites are among the most common type of minerals on Earth. MHPs have many favorable features, including strong light absorption, tunable band gap, long charge carrier diffusion, and low manufacturing cost, that make them promising candidates for solar energy and optoelectronic applications.

In order to demonstrate the developed NAMD approach, we focus on the pristine all-inorganic $\mathrm{CsPbI}_{3}$ perovskite and the same system, in which a cesium atom is replaced by an iodine atom (Figure 1a,c). ${ }^{34-36}$ The projected density of states (DOS) is split into $\mathrm{Cs}, \mathrm{Pb}$, and I contributions (Figure $\mathrm{lb}, \mathrm{d}$ ). The valence band minimum (VBM) and conduction band maximum (CBM) of pristine $\mathrm{CsPbI}_{3}$ are separated by a wide direct bandgap of $1.67 \mathrm{eV}$. The defect creates a trap state inside the bandgap, separated from the VBM and the CBM by 1.27 and $0.15 \mathrm{eV}$, respectively. The state is empty, and therefore, it acts as an electron trap. In the pristine system, the VBM is supported by both $\mathrm{Pb}$ and I atoms (Figure S2a), and the CBM is localized primarily on $\mathrm{Pb}$ atoms (Figure $\mathrm{S} 2 \mathrm{~b}$ ). In the defect
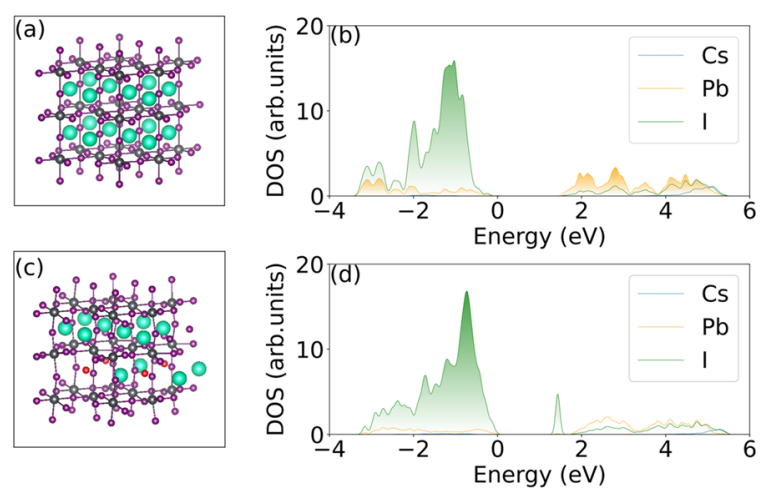

Figure 1. Geometric structures of (a) pristine $\mathrm{CsPbI}_{3}$ and (c) $\mathrm{CsPbI}_{3}$ with a cesium atom replaced by an iodine, indicated by the red ball. (b, d) Corresponding projected DOS. The zero energy is set to the VBM. The defect creates a midgap electron trap.

system, both the VBM and the CBM are supported by $\mathrm{Pb}$ and I (Figure S2c,e), and the trap state is supported by I atoms (Figure S2d), which agrees with the defect DOS (Figure 1d). The trap state is delocalized over many atoms, allowing it to couple well to free charge carriers.

In order to develop an accurate ML model, it is essential to describe accurately the system structure while keeping a balance between coverage of structural details and redundancy. Many studies have been performed on extracting structural features from molecules and bulk materials. ${ }^{13,14}$ For molecules, Rogers and Hahn proposed ${ }^{37}$ extended-connectivity fingerprints using a circular topological method that records the neighborhood of each non-hydrogen atom into multiple circular layers, up to a given diameter. Although the redundancy of the features is controllable, binary features are given, and radial information is not considered. For bulk systems, Behler and Parrinello presented ${ }^{38}$ a symmetry function that integrates radial information surrounding each atom with a given cutoff diameter into a real-valued number. The symmetry function is applicable to the optimized structure and considers the radial information to improve the quality of an extracted feature. Smith, Isayev and Roitberg et al. modified $^{39}$ heavily the Behler and Parrinello symmetry function. In addition to the radial relationship, angular information is also taken into account, increasing coverage of structural details while maintaining the size of the description. A good representation of the investigated structure is highly important when obvious relationships exist between structure and properties.

ANN is a widely used ML model that has been applied to predict thermodynamic stability, potential energy, formation energy, and other properties of perovskites and many other materials. ${ }^{40-43}$ The ANN architecture contains an input layer receiving a vector of features of a given length, several hidden layers which conduct linear transformation on the input followed by application of a nonlinear activation function, and lastly, an output layer giving the prediction, Figure S1. In the present work, the features are calculated using the modified symmetry function with the following form:

$$
\begin{aligned}
\mathrm{G}_{\mathrm{i}}^{\mathrm{mod}}= & 2^{1-\zeta} \sum_{j, k \neq \mathrm{i}}^{\text {atoms }}\left(1+\cos \left(\theta_{i j k}-\theta_{\mathrm{s}}\right)\right)^{\zeta} \times \mathrm{e}^{\left[-\eta\left(\frac{R_{i j}+R_{i k}}{2}-R_{\mathrm{s}}\right)^{2}\right]} \\
& \times f_{\mathrm{C}}\left(R_{\mathrm{ij} j}\right) f_{\mathrm{C}}\left(R_{i k}\right)
\end{aligned}
$$


The feature is divided into cosine and Gaussian terms, which include angular and radial information on atom $i$, respectively. $\theta_{i j k}$ is the angle between three atoms, with $i$ indicating the central atom. $\zeta$ controls the magnitude, and angle $\theta_{\mathrm{s}}$ defines the center of the angular term. $R_{i j}$ is the distance between atoms $i$ and $j$. Similarly to $\theta_{s}, R_{s}$ defines the center the Gaussian. $f_{\mathrm{C}}(R)$ is the cutoff function which limits the effective diameters and reduces computational effort:

$$
f_{C}(R)=\left\{\begin{array}{cc}
0.5 \times \cos \left(\frac{\pi R_{i j}}{R_{C}}\right)+0.5 & \text { for } R_{i j} \leq R_{C} \\
0.0 & \text { for } R_{i j}>R_{C}
\end{array}\right.
$$

The cutoff radius $R_{C}$ is set to $9.1 \AA$, which is the distance between the center of the rectangular simulation cell and its vertex. The NAMD calculations are performed using periodic boundary conditions, and the minimal image convention for the descriptors is used. The input layer passes the vector to all the neurons in the first hidden layer, where a linear transformation is applied to the features followed by a nonlinear activation function. Then, the values are passed to the next hidden layer. The same process is repeated several times, depending on the number of hidden layers, and finally the output layer receives the processed values, conducts a linear summation, and gives the prediction. The weights for the linear transformations are tuned during the learning process.

The values $\zeta$ and $\eta$ are set to 1 and 0.15 , respectively, to keep the radial and angular terms in eq 1 at similar magnitudes. These parameter choices ensure that all possible distance and angle values can be properly represented and distinguished. $R_{\mathrm{s}}$ and $\theta_{\mathrm{s}}$ are chosen to be the average distances and angles of each pair of elements in $\mathrm{CsPbI}_{3}$. There are three $R_{\mathrm{s}}$ and six $\theta_{\mathrm{s}}$ values for each element. The vector of features contains 72 real-valued features for the cesium and lead atoms, and 216 features for iodines. Thus, there are 360 features for each structure in total. The same features are used to train the models for predicting the energy gaps and NAC. However, because of the more complex dependence of NAC on system geometry, four hidden layers are used for NAC, while only two hidden layers are used for energy gaps. The number of neurons in the first and second hidden layers for energy gaps is 20 and 30 , respectively. The number of neurons in the four hidden layers used to train NAC is 20,30, 30 and 20, as shown in Figure S1.

The ab initio calculations are performed using the VASP software ${ }^{44-46}$ and the PBE functional. ${ }^{47}$ The structures shown in Figure 1 are optimized, heated up, and equilibrated at room temperature. Then, 7 ps trajectories are generated with a 1 fs time step in the microcanonical ensemble. $10 \%, 5 \%$, or $2.5 \%$ of the data are chosen, equally spaced along the trajectory, for training and validation, while the remaining data are used for testing. Below we focus in detail on the most stringent case, while the remaining results are summarized in Tables 1 and S1. Specifically, $2.5 \%$ represents 175 data points from the total of 7000 fs timesteps. $2 \%$, or 140 points, are used for training, while $0.5 \%$, or 35 points, are used for validation. $97.5 \%$, or 6825 points, are used for testing. The $140+35$ points used for training and validation are spaced every $40 \mathrm{fs}$. Every four of these points form the training set, and every fifth point is included in the validation set, i.e., $20 \mathrm{fs}, 100 \mathrm{fs}, 140 \mathrm{fs}, 180 \mathrm{fs}$, $220 \mathrm{fs}, 300 \mathrm{fs}, 340 \mathrm{fs}, 380 \mathrm{fs}, 420 \mathrm{fs}, 500 \mathrm{fs}$, etc. from the training set, while $60 \mathrm{fs}, 260 \mathrm{fs}, 460 \mathrm{fs}$, etc. form the validation
Table 1. Average Energy Gap, Average Absolute NAC, Root Mean Square NAC, Pure-Dephasing Time, and NAMD Transition Time of the Ab Initio and ANN Models for the Pristine System

\begin{tabular}{lcccc} 
& ab initio & $8 \% \mathrm{NN}$ & $4 \% \mathrm{NN}$ & $2 \% \mathrm{NN}$ \\
gap (eV) & 1.888 & 1.886 & 1.890 & 1.886 \\
Abs NAC (meV) & 0.448 & 0.450 & 0.475 & 0.479 \\
RMS NAC (meV) & 0.538 & 0.543 & 0.564 & 0.573 \\
pure-dephasing time (fs) & 8.493 & 8.500 & 8.905 & 8.554 \\
NAMD time (ns) & 89.27 & 98.78 & 81.73 & 83.22 \\
\hline
\end{tabular}

set. Reducing the training set from $2 \%$ to $1 \%$ allows us to obtain good results for the energy gaps, but the quality of the NAC prediction drops significantly.

All ANN training, validating, and testing are done with the Scikit-Learn package ${ }^{48}$ using the Python language. While training, the hyperbolic tangent function and the Adam optimizer are used as the activation function and the optimization algorithm. The L2 penalty is set to 0.005 and 0.0009 for the pristine and defect systems, respectively. Both models are trained with an adaptive learning rate starting from 0.001. The results are shown in Figures 2 and 3, and Tables 1 and $\mathrm{S} 1$.

Figure 2 shows the ab initio and ANN predicted energy gaps between the VBM, the CBM, and the trap state in the pristine and defect systems, with only $2 \%$ of the data used for training. Such a small fraction of the data allows us to develop and train ANN models showing excellent agreement with the ab initio calculations for both pristine and defective $\mathrm{CsPbI}_{3}$. Although the training data points, marked as yellow dots, do not cover all peaks and fluctuations, the testing set still fits well. In Figure $2 c$, we notice that the ANN model performs well most of the time, but the errors are relatively large in the beginning. That is because the first 20 data points are not sampled in the training set, and the model did not learn from these points. As the structures are getting close to the first training point, the error rapidly decreases to the average level. The larger error for the very early part of the trajectory not included in the training indicates that MD of the system is complex, and that $7000 \mathrm{fs}$ of $\mathrm{MD}$ is not sufficient to sample the entire space of relevant system geometries. That is why attempting to predict (extrapolate) the NAMD Hamiltonian in these systems based on a 7000 fs trajectory will produce unsatisfactory results. In contrast, the interpolation strategy developed here works very well, providing large computational savings.

The mean squared errors (MSE) are $0.164 \mathrm{meV}$ for the VBM-CBM bandgap in pristine $\mathrm{CsPbI}_{3}$, and $0.419,0.484$, and $0.211 \mathrm{meV}$ for the trap-VBM, CBM-trap and CBM-VBM gaps in defective $\mathrm{CsPbI}_{3}$. The errors are very small compared to the $1.67 \mathrm{eV}$ bandgap, indicating that the developed models have learned the relationship between the structure and bandgap, and are capable of accurate prediction.

The NAC has a more complex dependence on system geometry than the energy gaps. In particular, it exhibits a larger number of fast fluctuations between maxima and minima compared to the energy gaps. It is challenging to keep balance between underfitting and overfitting. We choose to develop the model as a smooth predictor which reasonably well learns fast fluctuation meanwhile reproducing most peaks. The peaks in the NAC are particularly important since the transition rate is proportional to the NAC squared and is the largest when the NAC peaks. The same activation function and optimizer are 

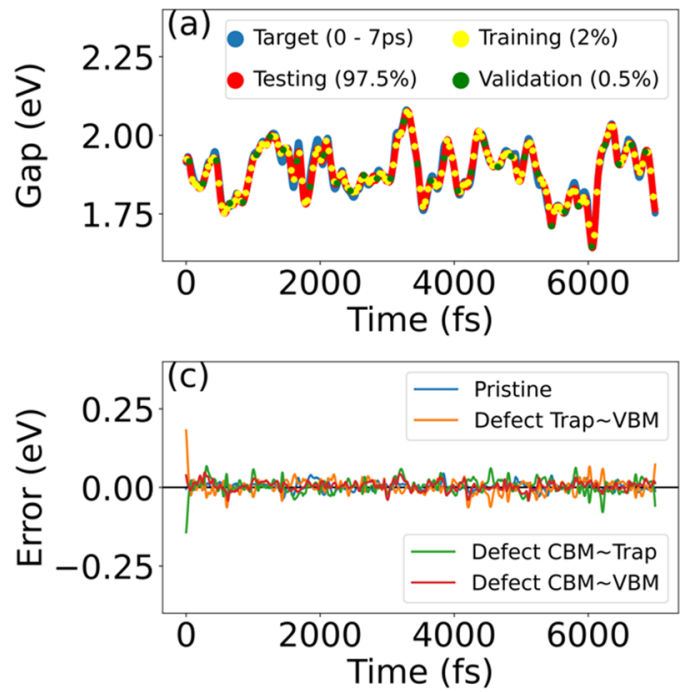

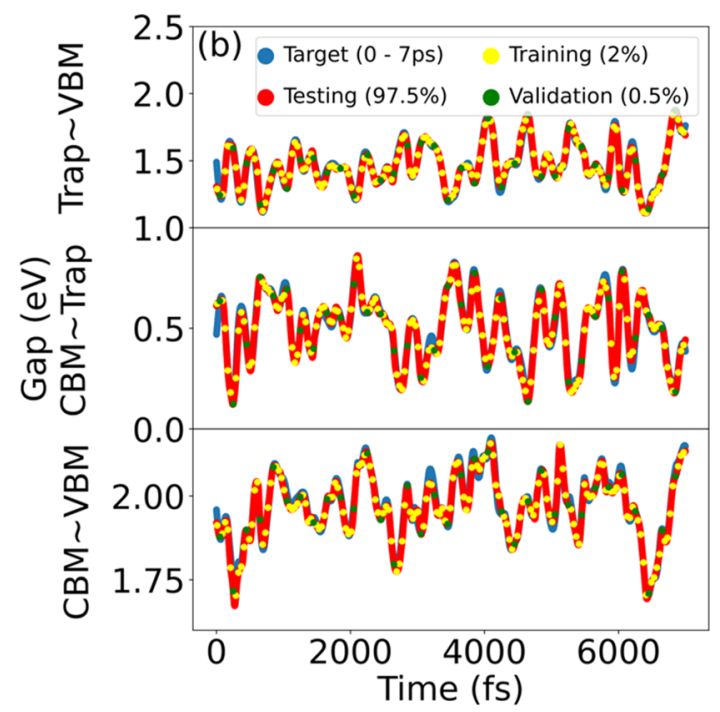

Figure 2. Energy gaps calculated with the ab initio and ANN models. ( $a, b)$ Gaps of the pristine and defect system, respectively. (c) ANN errors. The data are divided into training, validation, and testing sets, which contain $2 \%, 0.5 \%$, and $97.5 \%$ data points, respectively. The same ANN model accounts in (b) for gaps between trap and VBM, CBM and trap, and CBM and VBM. The errors are calculated as differences between the ab initio values and the ANN predictions.
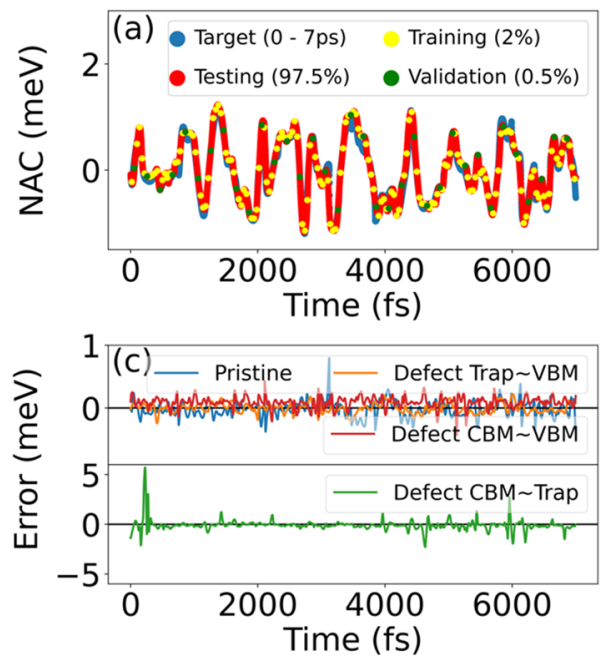

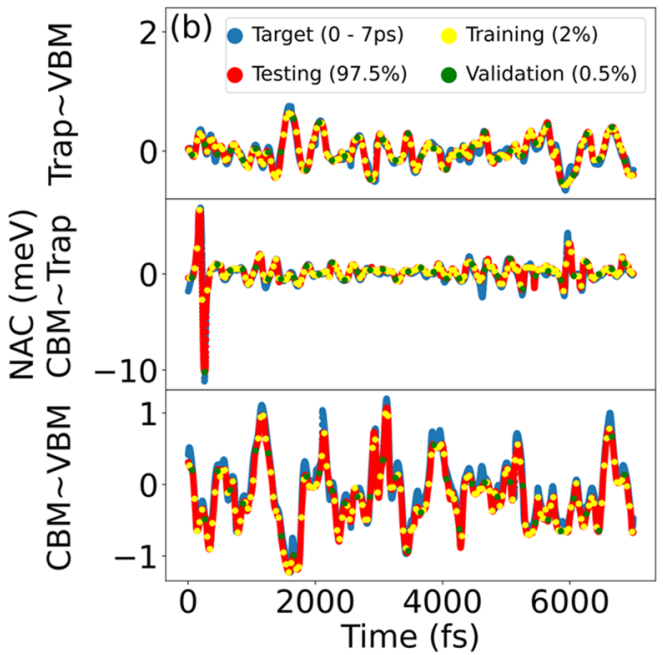

Figure 3. NAC calculated with the ab initio and ANN models. (a, b) NAC of the pristine and defect system, respectively. (c) ANN errors. The data are divided into training, validation, and testing sets, which contain $2 \%, 0.5 \%$, and $97.5 \%$ data points, respectively. Different ANN models account in (b) for the NAC between trap and VBM, CBM, and trap, and CBM and VBM. The errors are calculated as differences between the ab initio values and the ANN predictions.

used for the NAC as for the bandgaps. The L2 penalty is set to $1 \times 10^{-7}$ (pristine), $5 \times 10^{-8}$ (defect trap-VBM), $4 \times 10^{-8}$ (defect trap-CBM), and $1 \times 10^{-8}$ (defect CBM-VBM). Three independent ANN models are developed for defective $\mathrm{CsPbI}_{3}$ because of significant differences between them and the NAC complexity. The ANN models are able to predict the NAC accurately, as shown in Figure 3. The error for the NAC between the CBM and trap in the defect system is large at several special time steps (Figure 3c). That is because the NAC has large jumps that happen in a very short time, making the NAC magnitude 10 times larger than the average. Typically, this happens when the corresponding energy gap approaches 0 (Figure 2b). In turn, the errors at such points are relatively larger than at the other points. Shown in the middle panel of Figure $3 \mathrm{~b}$, few data points are sampled for the big jump in the training set (yellow dots). However, the ANN model still gives good predictions in such extreme situations. The MSEs are
$0.0142 \mathrm{meV}$ (pristine), $0.0058 \mathrm{meV}$ (defect Trap-VBM), $0.3087 \mathrm{meV}$ (defect trap-CBM), $0.0152 \mathrm{meV}$ (defect CBMVBM), and are small compared to the NAC values (Tables 1 and S1), indicating the models are good. Even the MSE of the defect NAC between Trap and CBM is reasonable compared to the NAC magnitude.

Fourier transforms of the energy gaps and NAC provide additional insight into how ANN performs with the perovskites. The FTs obtained from the ab initio and ANN data are compared in Figure 4. Overall, the NAC exhibits a broader range of frequencies than the energy gaps, and the defect system has more frequencies than the pristine system. Because $\mathrm{CsPbI}_{3}$ is composed of heavy elements, the FT spectra exhibit low frequencies. The largest peaks are around $50 \mathrm{~cm}^{-1}$ with strong signals extending to $200 \mathrm{~cm}^{-1}$ and minor peaks seen all the way to $400 \mathrm{~cm}^{-1}$, in particular, in Figure $4 \mathrm{f}$. Although the ANN reproduces all key features, some disagreement is seen 

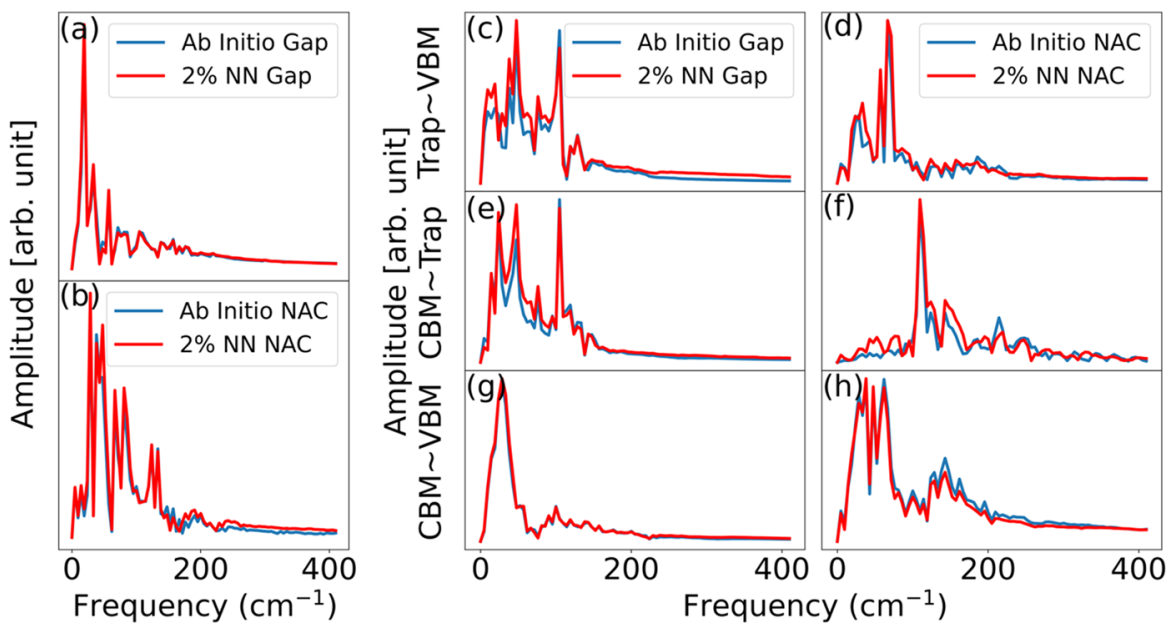

Figure 4. Fourier transforms of the ab initio and ANN values of the NAC and the energy gaps in (a, b) pristine $\mathrm{CsPbI}$ and (c-h) the defect system. The FT of the ANN data show good agreement with the ab initio FTs.
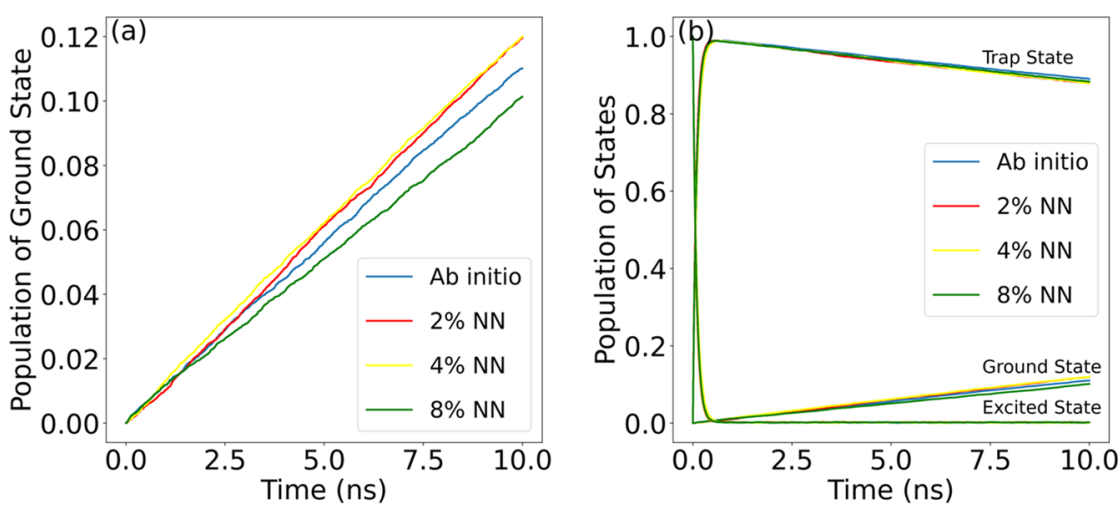

Figure 5. NAMD results of (a) pristine $\mathrm{CsPbI}_{3}$ and (b) the defect system. Shown are populations of the ground, trap, and excited states. ANNs with $2 \%, 4 \%$, and $8 \%$ of data used for training show comparable results, in good agreement with the ab initio data.

for the defect system in both the NAC and the energy gaps. That is because there are some peaks and fast fluctuations that are not reproduced exactly since our models are smooth predictors, which are expected to lose some precision.

It is instructive to consider why using $2 \%$ of data for the training gives good results for both the energy gaps and the NAC, while reducing the sampling to $1 \%$ creates problems for NAC training. Even sampling of $2.5 \%$ from 7000 points implies that a point every $40 \mathrm{fs}$ is used. $0.5 \%$ of the points are not used in the training and are included into the validation set. Because of the adopted protocol, the training set has gaps that are $80 \mathrm{fs}$ long. The $80 \mathrm{fs}$ period corresponds to the $417 \mathrm{~cm}^{-1}$ frequency. The NAC FT, Figure 4f, shows that $400 \mathrm{~cm}^{-1}$ is where the signals finally decay. Therefore, by performing an FT of the property to be trained, one can anticipate the fraction of the data points needed for the training. It is important to note that even though the NAC FT shows no signals above $400 \mathrm{~cm}^{-1}$, a large time step cannot be used in the numerical NAC calculation. $^{49-51}$ The NAC is an off-diagonal matrix element of the force operator, and therefore, it draws many analogies with the force. However, compared to the ground-state force, it is more sensitive to the nuclear geometry. Therefore, the time step used for the NAC calculation cannot be larger than the time step used to perform MD. One fs is a typical time step for MD simulations for various materials, such as the perovskites considered here. The ability to perform ab initio NAC calculations every 40th point along the MD trajectory provides large computational savings, more than 1 order of magnitude.

Figure 5 shows the NAMD results for the pristine and defective $\mathrm{CsPbI}_{3}$. The population of the ground state of the pristine $\mathrm{CsPbI}_{3}$ calculated ab initio increases from 0 to 0.11 in 10 ns. This implies an $11 \%$ probability of nonradiative electron-hole recombination within 10 ns. The NAMD calculations based on the ANN input give similar results. The populations obtained using the ANN trained with $2 \%$ and $4 \%$ of the data are 0.12 , while $8 \%$ ANN gives 0.10 at 10 ns. All ANN models generate good predictions, but the amount of the training data does not affect the final results much, which suggests that adding more than $2 \%$ of the training data does not help but costs more computational resources. Similar results are obtained in the defect system. All ANN-based populations follow the ab initio calculations.

Tables 1 and S1 summarize the ANN and ab initio results for the pristine and defect systems, including the canonically averaged energy gap and absolute NAC, the root mean square (RMS) NAC, the pure-dephasing times, and the nonradiative transition times. The pure-dephasing times are computed from energy gap fluctuations using the second-order cumulant approximation to the optical response function. ${ }^{52,53}$ The nonradiative transition times are obtained by fitting the data in Figure 5 to exponential functions or their short-time linear approximations, $P(t)=\exp (-t / \tau) \approx 1-t / \tau$, modified to reflect either rise or decay of a particular population. Considering the 
pristine system (Table 1), we observe that the ANN bandgaps can be slightly larger or smaller than the ab initio values. The NACs are slightly overestimated, which is a positive conclusion, because the main concern in predicting the NAC is to miss its maxima or minima that contribute most to the nonradiative relaxation. The ANN pure-dephasing times are slightly longer than the $a b$ initio value. This is because the pure-dephasing time depends on the energy gap fluctuation, ${ }^{53}$ and the ANN models smooth fluctuations out. The ANN numbers for the defect system (Table S1) can be both slightly larger and slightly smaller than the corresponding ab initio data.

In summary, we have developed a ML methodology that allows one to reduce the cost of ab initio NAMD calculations under the CPA by over an order of magnitude. We have noted that utilizing ML to predict evolution of the NA Hamiltonian constitutes a challenging task because excitation energies and in particular the NAC are complicated functions of nuclear geometries, compared to ground-state energies and forces. Therefore, we propose to take advantage of the extensively developed techniques that use $\mathrm{ML}$ to obtain ground-state forces and MD trajectories, sample the ab initio NA Hamiltonian along such trajectories, and interpolate between the sampled points. By applying the interpolation idea to pristine and defective MHPs, we have demonstrated that $2 \%$ of $\mathrm{ab}$ initio sampling is sufficient to get reliable NAMD results, providing $\sim 50$-fold computational savings. We note that problems in physical sciences differ from the majority of the problems treated with ML in other fields, such as time-series analysis of financial markets, sales prediction, etc. Most problems in physical sciences can be solved exactly in principle, however, at a high computational expense, while the problems treated by $\mathrm{ML}$ in other fields cannot be solved exactly in the foreseeable future. We have demonstrated that one can apply ML to predict simpler aspects of evolution of a physical system, i.e., the ground-state trajectory using a ML FF, and then sample more complex properties, i.e., the NAC, based on such prediction, and interpolate rather than extrapolate them. The strategy provides very accurate results at a low training cost.

\section{ASSOCIATED CONTENT}

\section{(s) Supporting Information}

The Supporting Information is available free of charge at https://pubs.acs.org/doi/10.1021/acs.jpclett.1c01645.

Schematic of the artificial neural networks used, charge densities of the key electronic states, results table for the defect system (PDF)

Raw data, including system geometries, energy gaps and nonadiabatic couplings (ZIP)

\section{AUTHOR INFORMATION}

\section{Corresponding Author}

Oleg V. Prezhdo - Department of Chemical Engineering and Department of Chemistry, University of Southern California, Los Angeles, California 90089, United States; (1) orcid.org/ 0000-0002-5140-7500; Email: prezhdo@usc.edu

\footnotetext{
Authors

Bipeng Wang - Department of Chemical Engineering, University of Southern California, Los Angeles, California 90089, United States; 이이이.org/0000-0003-0924-5867
}

Weibin Chu - Department of Chemistry, University of Southern California, Los Angeles, California 90089, United States; (1) orcid.org/0000-0001-5951-0337

Alexandre Tkatchenko - Department of Physics and Materials Science, University of Luxembourg, L-1511 Luxembourg, Luxembourg; (1) orcid.org/0000-0002-10124854

Complete contact information is available at: https://pubs.acs.org/10.1021/acs.jpclett.1c01645

\section{Notes}

The authors declare no competing financial interest.

\section{ACKNOWLEDGMENTS}

The work was supported by the U.S. National Science Foundation, Grant No. CHE-1900510.

\section{REFERENCES}

(1) Worth, G. A.; Cederbaum, L. S. Beyond Born-Oppenheimer: Molecular Dynamics through a Conical Intersection. Annu. Rev. Phys. Chem. 2004, 55, 127-158.

(2) Barbatti, M. Nonadiabatic Dynamics with Trajectory Surface Hopping Method. Wiley Interdisciplinary Reviews-Computational Molecular Science 2011, 1, 620-633.

(3) Wang, L. J.; Akimov, A.; Prezhdo, O. V. Recent Progress in Surface Hopping: 2011-2015. J. Phys. Chem. Lett. 2016, 7, 21002112.

(4) Stolow, A.; Bragg, A. E.; Neumark, D. M. Femtosecond TimeResolved Photoelectron Spectroscopy. Chem. Rev. 2004, 104, 17191757.

(5) Bakulin, A. A.; Selig, O.; Bakker, H. J.; Rezus, Y. L. A.; Muller, C.; Glaser, T.; Lovrincic, R.; Sun, Z. H.; Chen, Z. Y.; Walsh, A.; et al. Real-Time Observation of Organic Cation Reorientation in Methylammonium Lead Iodide Perovskites. J. Phys. Chem. Lett. 2015, 6, 3663-3669.

(6) Wright, A. D.; Buizza, L. R. V.; Savill, K. J.; Longo, G.; Snaith, H. J.; Johnston, M. B.; Herz, L. M. Ultrafast Excited-State Localization in Cs2AgBiBr6 Double Perovskite. J. Phys. Chem. Lett. 2021, 12, 33523360 .

(7) Akimov, A. V.; Prezhdo, O. V. The Pyxaid Program for NonAdiabatic Molecular Dynamics in Condensed Matter Systems. J. Chem. Theory Comput. 2013, 9, 4959-4972.

(8) Nijjar, P.; Jankowska, J.; Prezhdo, O. V. Ehrenfest and Classical Path Dynamics with Decoherence and Detailed Balance. J. Chem. Phys. 2019, 150, 204124.

(9) Zhou, G. Q.; Lu, G.; Prezhdo, O. V. Modeling Auger Processes with Nonadiabatic Molecular Dynamics. Nano Lett. 2021, 21, 756761.

(10) Zhu, H. M.; Miyata, K.; Fu, Y. P.; Wang, J.; Joshi, P. P.; Niesner, D.; Williams, K. W.; Jin, S.; Zhu, X. Y. Screening in Crystalline Liquids Protects Energetic Carriers in Hybrid Perovskites. Science 2016, 353, 1409-1413.

(11) Tong, C. J.; Li, L. Q.; Liu, L. M.; Prezhdo, O. V. Synergy between Ion Migration and Charge Carrier Recombination in MetalHalide Perovskites. J. Am. Chem. Soc. 2020, 142, 3060-3068.

(12) van Duin, A. C. T.; Dasgupta, S.; Lorant, F.; Goddard, W. A. Reaxff: A Reactive Force Field for Hydrocarbons. J. Phys. Chem. A 2001, 105, 9396-9409.

(13) Hansen, K.; Biegler, F.; Ramakrishnan, R.; Pronobis, W.; von Lilienfeld, O. A.; Muller, K. R.; Tkatchenko, A. Machine Learning Predictions of Molecular Properties: Accurate Many-Body Potentials and Nonlocality in Chemical Space. J. Phys. Chem. Lett. 2015, 6, 2326-2331.

(14) Unke, O. T.; Chmiela, S.; Sauceda, H. E.; Gastegger, M.; Poltavsky, I.; Schütt, K. T.; Tkatchenko, A.; Müller, K.-R. Machine Learning Force Fields. Chem. Rev. 2021, 121, DOI: 10.1021/ acs.chemrev.0c01111. 
(15) Ramakrishnan, R.; Hartmann, M.; Tapavicza, E.; von Lilienfeld, O. A. Electronic Spectra from TDDFT and Machine Learning in Chemical Space. J. Chem. Phys. 2015, 143, 084111.

(16) Pronobis, W.; Schutt, K. T.; Tkatchenko, A.; Muller, K. R. Capturing Intensive and Extensive DFT/TDDFT Molecular Properties with Machine Learning. Eur. Phys. J. B 2018, 91, 178.

(17) Ben Mahmoud, C.; Anelli, A.; Csanyi, G.; Ceriotti, M. Learning the Electronic Density of States in Condensed Matter. Phys. Rev. B: Condens. Matter Mater. Phys. 2020, 102, 235130.

(18) Zhang, Z. S.; Zhang, Y.; Wang, J. Z.; Xu, J. Z.; Long, R. DopingInduced Charge Localization Suppresses Electron-Hole Recombination in Copper Zinc Tin Sulfide: Quantum Dynamics Combined with Deep Neural Networks Analysis. J. Phys. Chem. Lett. 2021, 12, 835842.

(19) Guan, Y. F.; Yarkony, D. R. Accurate Neural Network Representation of the Ab Initio Determined Spin-Orbit Interaction in the Diabatic Representation Including the Effects of Conical Intersections. J. Phys. Chem. Lett. 2020, 11, 1848-1858.

(20) Chen, W. K.; Liu, X. Y.; Fang, W. H.; Dral, P. O.; Cui, G. L. Deep Learning for Nonadiabatic Excited-State Dynamics. J. Phys. Chem. Lett. 2018, 9, 6702-6708.

(21) Sonoda, S.; Murata, N. Neural Network with Unbounded Activation Functions Is Universal Approximator. Applied and Computational Harmonic Analysis 2017, 43, 233-268.

(22) Chaban, V. V.; Prezhdo, V. V.; Prezhdo, O. V. Covalent Linking Greatly Enhances Photoinduced Electron Transfer in FullereneQuantum Dot Nanocomposites: Time-Domain Ab Initio Study. J. Phys. Chem. Lett. 2013, 4, 1-6.

(23) Li, L. Q.; Long, R.; Prezhdo, O. V. Charge Separation and Recombination in Two-Dimensional Mos2/Ws2: Time-Domain Ab Initio Modeling. Chem. Mater. 2017, 29, 2466-2473.

(24) Long, R.; Casanova, D.; Fang, W. H.; Prezhdo, O. V. Donor Acceptor Interaction Determines the Mechanism of Photoinduced Electron Injection from Graphene Quantum Dots into Tio2:PiStacking Supersedes Covalent Bonding. J. Am. Chem. Soc. 2017, 139, 2619-2629.

(25) He, J. L.; Vasenko, A. S.; Long, R.; Prezhdo, O. V. Halide Composition Controls Electron-Hole Recombination in Cesium-Lead Halide Perovskite Quantum Dots: A Time Domain Ab Lnitio Study. J. Phys. Chem. Lett. 2018, 9, 1872-1879.

(26) Li, W.; Vasenko, A. S.; Tang, J. F.; Prezhdo, O. V. Anharmonicity Extends Carrier Lifetimes in Lead Halide Perovskites at Elevated Temperatures. J. Phys. Chem. Lett. 2019, 10, 6219-6226. (27) Shi, R.; Vasenko, A. S.; Long, R.; Prezhdo, O. V. Edge Influence on Charge Carrier Localization and Lifetime in Ch3nh3pbbr3 Perovskite: Ab Initio Quantum Dynamics Simulation. J. Phys. Chem. Lett. 2020, 11, 9100-9109.

(28) Qiao, L.; Fang, W. H.; Long, R.; Prezhdo, O. V. Atomic Model for Alkali Metal Passivation of Point Defects at Perovskite Grain Boundaries. ACS Energy Letters 2020, 5, 3813-3820.

(29) Wang, Z. L.; Altmann, P.; Gadermaier, C.; Yang, Y. T.; Li, W.; Ghirardini, L.; Trovatello, C.; Finazzi, M.; Duo, L.; Celebrano, M.; Long, R.; Akinwande, D.; Prezhdo, O. V.; Cerullo, G.; Dal Conte, S. Phonon-Mediated Interlayer Charge Separation and Recombination in a Mose2/Wse2 Heterostructure. Nano Lett. 2021, 21, 2165-2173.

(30) Tomko, J. A.; Runnerstrom, E. L.; Wang, Y. S.; Chu, W. B.; Nolen, J. R.; Olson, D. H.; Kelley, K. P.; Cleri, A.; Nordlander, J.; Caldwell, J. D.; Prezhdo, O. V.; Maria, J. P.; Hopkins, P. E. LongLived Modulation of Plasmonic Absorption by Ballistic Thermal Injection. Nat. Nanotechnol. 2021, 16, 47-51.

(31) Kim, H.-S.; Lee, C.-R.; Im, J.-H.; Lee, K.-B.; Moehl, T.; Marchioro, A.; Moon, S.-J.; Humphry-Baker, R.; Yum, J.-H.; Moser, J. E.; Grätzel, M.; Park, N.-G. Lead Iodide Perovskite Sensitized AllSolid-State Submicron Thin Film Mesoscopic Solar Cell with Efficiency Exceeding 9\%. Sci. Rep. 2012, 2, 591.

(32) Lee, M. M.; Teuscher, J.; Miyasaka, T.; Murakami, T. N.; Snaith, H. J. Efficient Hybrid Solar Cells Based on Meso-Superstructured Organometal Halide Perovskites. Science 2012, 338, 643.
(33) Noel, N. K.; Stranks, S. D.; Abate, A.; Wehrenfennig, C.; Guarnera, S.; Haghighirad, A. A.; Sadhanala, A.; Eperon, G. E.; Pathak, S. K.; Johnston, M. B.; Petrozza, A.; Herz, L. M.; Snaith, H. J. Lead-Free Organic-Inorganic Tin Halide Perovskites for Photovoltaic Applications. Energy Environ. Sci. 2014, 7, 3061-3068.

(34) Chu, W.; Saidi, W. A.; Zhao, J.; Prezhdo, O. V. Soft Lattice and Defect Covalency Rationalize Tolerance of B-Cspbi3 Perovskite Solar Cells to Native Defects. Angew. Chem. Int. Ed. 2020, 59, 6435-6441, DOI: $10.1002 /$ anie.201915702.

(35) Wang, Y.; Dar, M. I.; Ono, L. K.; Zhang, T.; Kan, M.; Li, Y.; Zhang, L.; Wang, X.; Yang, Y.; Gao, X. Thermodynamically Stabilized B-Cspbi3-Based Perovskite Solar Cells with Efficiencies > 18\%. Science 2019, 365, 591-595, DOI: 10.1126/science.aav8680.

(36) Li, W.; She, Y. L.; Vasenko, A. S.; Prezhdo, O. V. Ab Initio Nonadiabatic Molecular Dynamics of Charge Carriers in Metal Halide Perovskites. Nanoscale 2021, 13, 10239-10265.

(37) Rogers, D.; Hahn, M. Extended-Connectivity Fingerprints. J. Chem. Inf. Model. 2010, 50, 742-754.

(38) Behler, J.; Parrinello, M. Generalized Neural-Network Representation of High-Dimensional Potential-Energy Surfaces. Phys. Rev. Lett. 2007, 98, 146401.

(39) Smith, J. S.; Isayev, O.; Roitberg, A. E. Ani-1: An Extensible Neural Network Potential with Dft Accuracy at Force Field Computational Cost. Chemical Science 2017, 8, 3192-3203.

(40) Cho, E. H.; Lin, L. C. Nanoporous Material Recognition Via 3d Convolutional Neural Networks: Prediction of Adsorption Properties. J. Phys. Chem. Lett. 2021, 12, 2279-2285.

(41) Lee, E. M. Y.; Ludwig, T.; Yu, B. Y.; Singh, A. R.; Gygi, F.; Norskov, J. K.; de Pablo, J. J. Neural Network Sampling of the Free Energy Landscape for Nitrogen Dissociation on Ruthenium. J. Phys. Chem. Lett. 2021, 12, 2954-2962.

(42) Secor, M.; Soudackov, A. V.; Hammes-Schiffer, S. Artificial Neural Networks as Mappings between Proton Potentials, Wave Functions, Densities, and Energy Levels. J. Phys. Chem. Lett. 2021, 12, 2206-2212.

(43) Tong, Q. C.; Gao, P. Y.; Liu, H. Y.; Xie, Y.; Lv, J.; Wang, Y. C.; Zhao, J. J. Combining Machine Learning Potential and Structure Prediction for Accelerated Materials Design and Discovery. J. Phys. Chem. Lett. 2020, 11, 8710-8720.

(44) Kresse, G.; Hafner, J. Ab Initio Molecular Dynamics for Liquid Metals. Phys. Rev. B: Condens. Matter Mater. Phys. 1993, 47, 558-561.

(45) Kresse, G.; Hafner, J. Ab Initio Molecular Dynamics for OpenShell Transition Metals. Phys. Rev. B: Condens. Matter Mater. Phys. 1993, 48, 13115-13118.

(46) Kresse, G.; Hafner, J. Ab Initio Molecular-Dynamics Simulation of the Liquid-Metal-Amorphous-Semiconductor Transition in Germanium. Phys. Rev. B: Condens. Matter Mater. Phys. 1994, 49, 14251-14269.

(47) Kresse, G.; Joubert, D. From Ultrasoft Pseudopotentials to the Projector Augmented-Wave Method. Phys. Rev. B: Condens. Matter Mater. Phys. 1999, 59, 1758-1775.

(48) Pedregosa, F.; Varoquaux, G.; Gramfort, A.; Michel, V.; Thirion, B.; Grisel, O.; Blondel, M.; Prettenhofer, P.; Weiss, R.; Dubourg, V. Scikit-Learn: Machine Learning in Python. J. Mach. Learn. Res. 2011, 12, 2825-2830.

(49) Hammes-Schiffer, S.; Tully, J. C. Proton-Transfer in Solution Molecular-Dynamics with Quantum Transitions. J. Chem. Phys. 1994, 101, 4657-4667.

(50) Chu, W. B.; Prezhdo, O. V. Concentric Approximation for Fast and Accurate Numerical Evaluation of Nonadiabatic Coupling with Projector Augmented-Wave Pseudopotentials. J. Phys. Chem. Lett. 2021, 12, 3082-3089.

(51) Chu, W. B.; Zheng, Q. J.; Akimov, A. V.; Zhao, J.; Saidi, W. A.; Prezhdo, O. V. Accurate Computation of Nonadiabatic Coupling with Projector Augmented-Wave Pseudopotentials. J. Phys. Chem. Lett. 2020, 11, 10073-10080.

(52) Habenicht, B. F.; Kamisaka, H.; Yamashita, K.; Prezhdo, O. V. $\mathrm{Ab}$ Initio Study of Vibrational Dephasing of Electronic Excitations in Semiconducting Carbon Nanotubes. Nano Lett. 2007, 7, 3260-3265. 
(53) Akimov, A. V.; Prezhdo, O. V. Persistent Electronic Coherence Despite Rapid Loss of Electron-Nuclear Correlation. J. Phys. Chem. Lett. 2013, 4, 3857-3864. 